\title{
Affectivités entre tempéraments et troubles
}

\author{
Gottfried R. S. Treviranus
}

Spécialiste en psychiatrie et psychothérapie, FMH

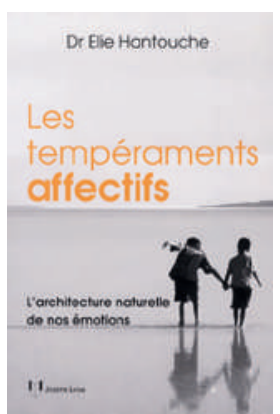

Elie Hantouche Les tempéraments affectifs

\section{L'architecture} naturelle de nos émotions

Paris: Josette Lyon; 2014.

379 pages. $34.10 \mathrm{CHF}$. ISBN 978-2-843-19319-4
En relatant pour nous une expérience de réflexions diagnostiques et thérapeutiques sur les tempéraments menée pendant 30 ans au cours de recherches sur les «tempéraments affectifs» du Libanais arménien Hagop Akiskal et sur le spectrum bipolaire (et unipolaire) de Jules Angst, le Libanais maronite parisien Elie Hantouche nous fait cadeau d'un livre exceptionnel destiné aux patients et au personnel traitant qui désirent se renseigner sur les questions structurelles brûlantes psychologiques et psychiatriques du domaine des troubles anxio-affectifs. Son admiration pour les maîtres-penseurs, incluant l'autre Libanais Elie Karam et le Grec Athanasios Koukopoulos, se conjugue avec la documentation du vaste travail de recherche et d'agrégation dont il est un des réalisateurs les plus actifs, sans disposer d'une fonction académique vraiment à hauteur de cette tâche énorme. L'utilité thérapeutique par l'emploi argumenté, souple et différencié des modulateurs d'humeur (souvent au lieu des antidépresseurs en règle générale risqués dans ce domaine) est immense, comme l'est l'approche acceptante et raisonnée face aux tempéraments. La méconnaissance de ces grands modulateurs de la vie psychique se révèle ici comme obstacle majeur à la gestion harmonieuse de soi (et non pas de l'autre), voire aux soins des troubles entrecroisés avec eux. Si en suivant Kurt Lewin vous cherchez le meilleur en pratique thérapeutique - le voilà: une bonne théorie!

\section{Evaluer les richesses de l'histoire des idées selon leur utilité clinique}

Ce livre, parmi la douzaine de ses autres titres cliniques, toujours accessibles au grand public, est le fruit, de nos jours plus systématique, de son insatisfaction intellectuelle inlassable et méticuleuse face d'abord aux problèmes résistants des atteints de troubles obsessionnels-compulsifs, au Centre hospitalier Sainte Anne et à la Salpêtrière et, depuis 2007, à ceux des plus de 3000 patients soignés par lui et son équipe au "CTAH» du VIII ${ }^{\mathrm{e}}$ de Paris.

Le livre, comme le CTAH, vous accueille avec le charme levantin d'une largesse qui déroule les richesses de l'histoire des idées depuis son origine hippocratique et galénique, en les évaluant toujours selon leur utilité clinique. Ainsi l'auteur, toujours stimulé par son intérêt thérapeutique-philosophique, développe l'essentiel sur les approches synergiques et contradictoires des définitions et modèles, aussi développementaux, concernant les tempéraments et les caractères, aboutissant ainsi sur les paramètres de la polarité, dominance, réactivité, intensité, stabilité voire rigidité et finalement synergie interactive dans la constitution des phénomènes affectifs dans l'individu et son identité. Constants dans tout cela sont ses efforts de médiation théorique de l'approche des "tempéraments affectifs» avec les courants hégémoniques psycho-analytique ou des attachements. Ceux-ci dans l'engrenage de l'approche d'appropriation minimalisent souvent l'évidence de préformations mentales innées, ou acquises, et leurs relations avec les véritables troubles. Moyennant les cinq schémas précoces maintenus de Jeffrey E. Young nous assistons à des extensions préliminaires dans les «scénarios» répétitifs de la vie psychique individuelle complexe comme rencontrée auprès de 45 cyclothymiques. A ce stade, nous sommes témoins comment Hantouche, qui dit bien aimer «le terme 'mouvement'

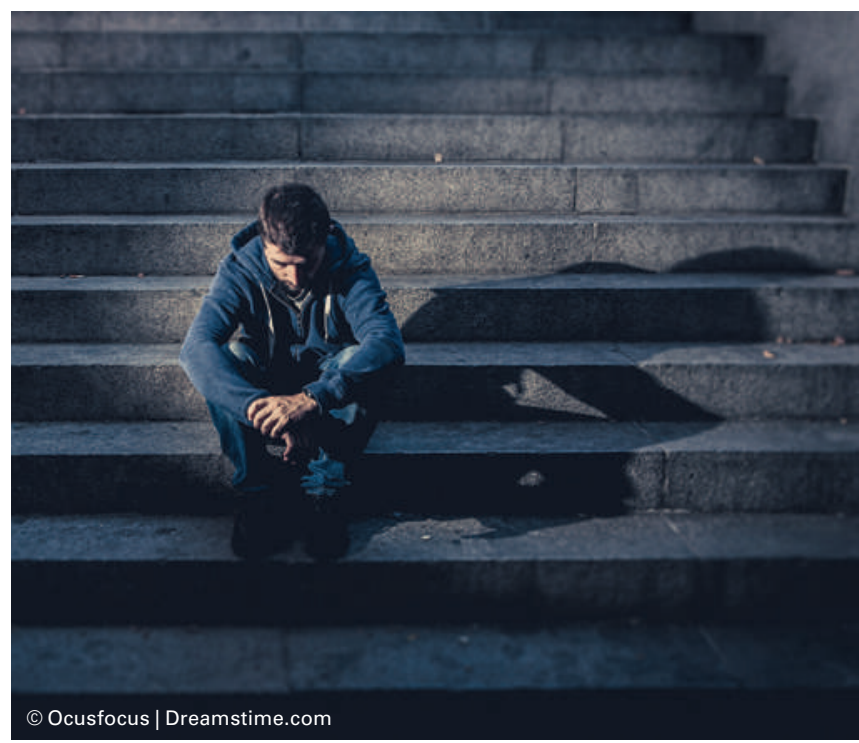

Le tempérament détermine la partie biologique de notre personnalité en termes de réactivité, de sensibilité et de stabilité émotionnelle. 
pour parler des émotions et des affects», passe à l'action scientifique moyennant l'outil globalement appliqué par le courant sous-dit TEMPS-Autoévaluation (env. 1995), conçu avec l'apport par ex. du prof. Andreas Marneros de Halle, et son propre questionnaire RIPOST (2010).

\section{Un travail de comparaison}

Loin de vous contraindre à utiliser des outils «à lui», il vous offre un grand choix de schémas et questionnaires autres permettant d'effectuer un véritable travail de comparaison. Dans le TEMPS-A, l'abandon du tempérament grec lymphatique (flegmatique), dont des personnalités languides et involontaires sont porteurs (et faisant pourtant bien partie du spectre de la maniacodépression), reste peu motivé, comme d'ailleurs le manque d'un tempérament «effrayé». Néanmoins, le succès du TEMPS-A a été considérable surtout par la facette cyclothymique qui s'est révélée - aussi par «les idées intenses qui font mal» moyennant «des blessures internes» - une véritable dimension de prédiction, dans laquelle l'individu peut s'enfoncer dans des troubles du spectre bipolaire toujours plus graves. Ceci est exposé par notre expert de pointe au travers des résultats épidémiologiques, qui lient les troubles avec des dimensions tempéramentales. Les données y soutiennent des interactions complexes entre tempéraments et troubles par stress traumatisant (borderline, suicidalité) en niant les causalités simples. De même une revue complète et à jour de publications montre l'impact énorme surtout, mais pas uniquement, du tempérament cyclothymique (et enfin les amalgames avec les dépressions ou le trouble bipolaire mature) sur d'autres troubles clefs de la psychiatrie: les troubles anxieux, les addictions chimiques (explorés par Andreas Erfurth) et comportementales, les troubles post-partum etc. D'autres chapitres magistraux s'entrecroisent: les valeurs adaptives sociétaires et évolutionnaires des tempéraments (affectifs) avec leurs attitudes envers l'incertain (avec l'omission regrettable de Richard M. Sorrentino) et leurs conséquences sur le privé des amours, sur la politique et sur le travail. Y brillent des passages lucides sur les bons paradoxes sous-jacents à la créativité, qui risquent par nécessité une connivence avec l'hyperthymie, voire mono-hypomanie chronique «ensoleillée» - malgré son revers irritable et «sombre».

\section{Toute théorie est temporelle}

Suivant certainement aussi l'avis du maître de la filière Jules Angst, il faut rappeler la temporalité de toute théorie. Comme membre marginal de ce mouvement depuis 2003, j'imagine aussi son dépassement par une rigueur intégrative accrue, qui se réclamerait de la véritable tridimensionnalité de la triade «Pensée-Action-Humeur» de la filière aliéniste. Celle-ci serait à complé-

\section{L'auteur développe l'essentiel sur les approches synergiques et contradictoires.}

ter au «nombre 4» - qui intrigue Hantouche - par la dimension du temps. On y trouverait des correspondances personnologiques chez Richard Depue, d'autres neuroanatomiques comme les circuits cortico-subcorticocorticaux conditionnant la séquence tempéramentale au long de ce que dans l'approche j'appellerais la vague appropriatrice.

En bref: si vous vous permettez de considérer une «architecture naturelle» agissant par soi-même d'en bas sur la pensée, l'action et l'humeur, voici l'introduction formidable d'un véritable expert basée sur un engagement thérapeutique toujours argumenté.

\section{Remerciement}

Gottfried R. S. Treviranus remercie à Anne-Marie Cuttat qui a rédigé le texte. 